\title{
The rate of electron-transfer reactions in the diffusive limit*
}

\author{
W.Schmickler \\ Abteilung Elektrochemie, University of Ulm, D-89069 Ulm, Germany
}

Received August 8, 2001, in final form October 10, 2001

\begin{abstract}
A simple electron-transfer reaction is treated in the diffusive limit, in which the motion of the solvent is governed by the Smoluchowski equation. The electronic transition probability is calculated from the Landau-Zener expression. The rate constant of the reaction is calculated as a function of the strength of the electronic interaction between the reactants. For weak interactions, the rate is the same as that obtained form first-order perturbation theory. For strong interactions, solvent dynamics is rate-determining. The calculations presented here bridge these two limits.
\end{abstract}

Key words: electron transfer reactions, diffusive limit

PACS: $82.30 . F, 82.20 . G k$

\section{Introduction}

Electron-transfer reactions play a major role in physics, chemistry, and biology alike [1]. Much of our present understanding of the way these reactions proceed in condensed phases is based on the works of Marcus [2] and Hush [3], which are based on classical statistical mechanics, and of Levich and Dogonadze [4], who employed first-order perturbation theory. These two classes of theories refer to different strengths of the electronic interaction between the reacting partners: Marcus and Hush consider the adiabatic limit, in which the interaction is strong, while the Levich and Dogonadze theory treats nonadiabatic reactions with weak electronic coupling.

Till the present day, despite many efforts (see e.g. [5-8]), the gap between the adiabatic and the nonadiabatic limits has never been quite closed. For the special case of electrochemical reactions Mohr and Schmickler [9] have recently derived an expression for the rate constant that is valid for all interaction strengths. However, solvent dynamics is not considered in this work, so that its validity is limited to timescales shorter than the solvent relaxation time.

In this work, we treat the simple case in which the electron transfer is coupled to classical solvent modes only, and in which solvent dynamics is overdamped. The

*Dedicated to Jean-Pierre Badiali on the occasion of his 60th birthday. 
motion of the system is then governed by the Smoluchowski equation, which we solve, using the electronic transition probability as a boundary condition. In this way we obtain an expression for the rate constant which is valid for arbitrary strengths of the electronic interaction.

\section{Diffusion controlled electron transfer}

We consider electron exchange between two reactants in a solution, and assume that this exchange is coupled to a classical bath. In this case, the system can be characterized by a single, effective bath coordinate $q$. Within the harmonic approximation the diabatic potential energy curves for the initial and final states are parabolas, which intersect at the saddle point $q_{a}$ (see figure 1), and which have their equilibrium positions at $q=0$ and $q=q_{f}$. Within this model, which originated with Marcus [2] and Hush [3], electron transfer is an activated process: A thermal fluctuation takes the system from the bottom of the initial well to the saddle point, where

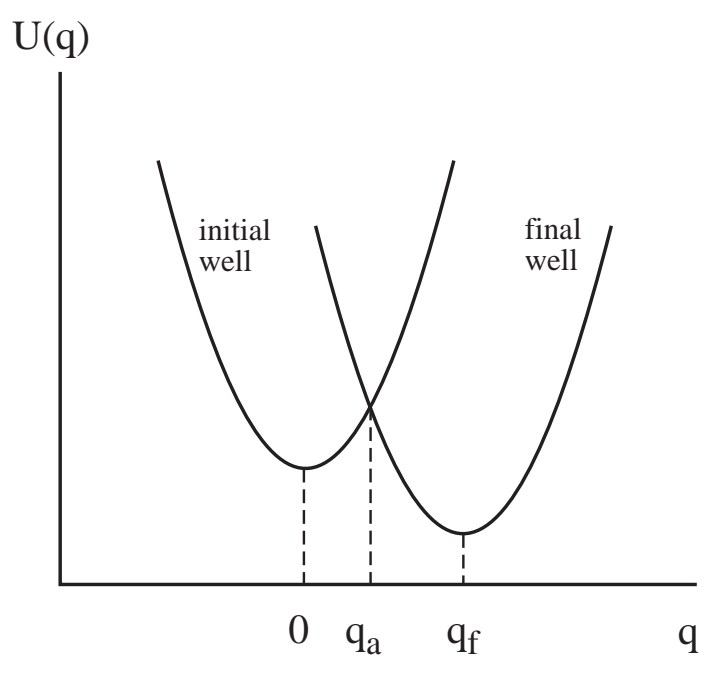

Figure 1. Potential energy curves for a simple electron-transfer reaction electron exchange may occur. The original papers by Marcus and Hush treated only the adiabatic limit, in which the electronic coupling between the reactants is so strong that an electron is exchanged every time the system passes the saddle point. Here we consider the general case, in which the coupling has an arbitrary strength, and the system may pass the saddle point without the occurrence of an electron transfer.

Before the reaction, the system undergoes a stochastic motion in the initial well. In the overdamped case, which we consider here, the probability $P(q, t)$ to find the system at position $q$ at the time $t$ obeys a Smoluchowski equation of the form [10]:

$$
\frac{\partial P(q, t)}{\partial t}=-\frac{\partial}{\partial q} j(q, t)=D \frac{\partial}{\partial q}\left[\frac{\partial P(q, t)}{\partial q}+\beta U_{i}^{\prime}(q) P(q, t)\right] .
$$

Here, $j(q, t)$ is the corresponding probability current, $D$ is the diffusion coefficient, which we take as constant, $\beta=1 / k_{\mathrm{B}} T, k_{\mathrm{B}}$ is Boltzmann's constant, $T$ the temperature, and $U_{i}(q)=\frac{1}{2} m \omega^{2} q^{2}$ the potential in the initial well. For numerical calculations we will set the effective mass $m$ and the frequency $\omega$ equal to unity.

At $q=q_{a}$ the system can exchange an electron and escape from the initial well. This results in a radiative boundary condition of the form:

$$
j\left(q_{a}, t\right)=-\kappa P\left(q_{a}, t\right)
$$


For the electronic transition probability $t(v)$ we use the Landau-Zener expression $[11,12]$ :

$$
t(v)=\frac{1-\exp (-2 \pi \nu)}{1-\frac{1}{2} \exp (-2 \pi \nu)} \quad \text { with } \quad \nu=\frac{|V|^{2}}{\hbar m \omega^{2}\left|q_{f} v\right|} .
$$

Here, $V$ is the matrix element for the electronic coupling, and $v$ is the velocity of the system near the saddle point. The denominator in the first part of equation (3) takes account of multiple crossing of the saddle point [1].

The Landau-Zener equation results in the following expression for the escape rate $\kappa$ :

$$
\kappa=\left(2 \pi k_{\mathrm{B}} T / m\right)^{(1 / 2)} \int_{0}^{\infty} \mathrm{d} v \exp \left(-\frac{m v^{2}}{2 k_{\mathrm{B}} T}\right) t(v) .
$$

The rate of electron transfer can be calculated as the inverse of the first mean passage time $\tau$, for which Szabo et al. [13] and Deutch [14] have obtained explicit expressions. For the case in which the initial distribution equals the equilibrium distribution, $P(q, 0)=p_{0}(q)$, the result is:

$$
\tau=\left[\kappa p_{0}(a)\right]^{-1}+I_{2}, \quad \text { with } \quad I_{2}=\frac{1}{D} \int_{q_{a}}^{\infty} \mathrm{d} q\left[p_{0}\right]^{-1}\left[\int_{q}^{\infty} \mathrm{d} x p_{0}(x)\right]^{2} .
$$

Note that the first term is just the inverse of the rate for the case of a small electronic transition rate $\kappa$, while the second term is independent of the electronic coupling. For the harmonic potential the integral $I_{2}$ can be simplified:

$$
I_{2}=\frac{2 \gamma}{\omega^{2}}\left\{\frac{\ln 2}{2}+\frac{\sqrt{\pi}}{4} \int_{0}^{\alpha q_{a}} \mathrm{~d} y \exp \left(y^{2}\right)[1+\operatorname{erf}(y)]^{2}\right\} \quad \text { with } \quad \alpha=\sqrt{\frac{m \omega^{2}}{2 k_{\mathrm{B}} T}}
$$

where we have introduced the friction coefficient $\gamma=k_{\mathrm{B}} T / m D$.

The remaining integration is easily performed numerically. The two limiting cases of weak and strong electronic interaction can be calculated explicitly. For weak coupling the first term in equation (5) dominates, and taking the appropriate limit of equation (4) gives for the rate constant:

$$
k=1 / \tau=\frac{|V|^{2}}{\hbar} \sqrt{\frac{\pi}{k_{\mathrm{B}} T \lambda}} \exp \left(-\beta E_{\mathrm{act}}\right),
$$

where $\lambda=m \omega^{2} x_{f}^{2} / 2$ is the energy of reorganization, and $E_{\text {act }}=m \omega^{2} x_{a}^{2} / 2$ the energy of activation. This expression is identical to that obtained by first order perturbation theory $[1,4]$.

For strong coupling, the first term in equation (5) is negligible. For a sufficiently high energy of activation, when $\beta E_{\text {act }} \gg 1$, the error function in equation (6) can be replaced by unity, and the integral can be replaced by its asymptotic value. The leading term is:

$$
k=\frac{\omega^{2}}{2 \pi \gamma} \sqrt{\pi \beta E_{\text {act }}} \exp -\beta E_{\text {act }}
$$

and is identical to the expression derived by Kramers $[15,16]$ for the case of a cuspshaped barrier. 
The general case has to be treated numerically. Figure 2 shows a plot of the rate constant versus the LandauZener parameter $\sigma=|V|^{2} / \hbar m \omega^{2} q_{f}$ and for three different values of the friction coefficient $\gamma$. For small interactions, the rate is proportional to the strengths $|V|^{2}$, while for higher interaction strengths, solvent dynamics becomes rate-determining, and the rate attains a constant value. The higher the coefficient of friction, the lower is the limiting rate for high $\sigma$. For very strong electronic interactions, the energy of activation is lowered, and the rate should rise again [17]. However, this effect is not considered here since we calculate the

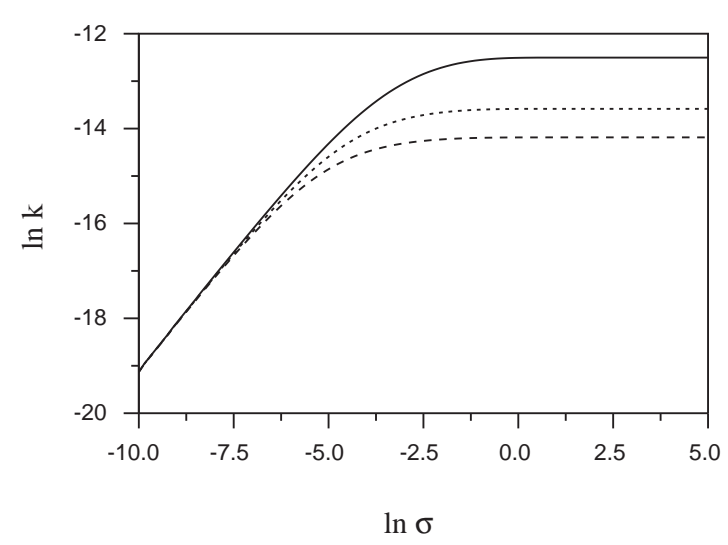

Figure 2. Rate constant versus the Landau-Zener parameter $\sigma$; full line: $\gamma / \omega=10$, dotted line: $\gamma / \omega=50$; dashed line $\gamma / \omega=100$. rate from the diabatic potential energy curves.

\section{Conclusion}

To the best of our knowledge, this is the first model that applies for all strengths of the electronic interaction between the reactants. It encompasses both the nonadiabatic and the adiabatic regions, and nicely illustrates the interplay between electron transfer and solvent dynamics. From equation (5) it can be seen that the rate constant $k$ obeys a relation of the form:

$$
\frac{1}{k}=\frac{1}{k_{\mathrm{el}}}+\frac{1}{k_{\mathrm{dyn}}}
$$

where $k_{\mathrm{el}}$ depends on the electronic interaction, and $k_{\mathrm{dyn}}$ on the solvent dynamics. An equation of this form is sometimes assumed as an interpolation formula between the adiabatic and the nonadiabatic limits $[18,19]$. Here this relation arises naturally from the solution of the diffusion equation. Note, however, that $k_{\mathrm{el}}$ goes beyond first-order perturbation theory.

Our model applies only to the simplest possible case, in which quantum modes play no role, and solvent dynamics are overdamped. It should be extendable to the case where the dynamics are not overdamped and obey a Fokker-Plank involving both the position $q$ and the velocity $v$. However, in this case the solution is likely to be wholly numerical.

\section{Acknowledgement}

Financial support by the Volkswagenstiftung is gratefully acknowledged. 


\section{References}

1. Kuznetsov A.M. Charge Transfer in Physics, Chemistry and Biology. Gordon \& Breach, 1995.

2. Marcus R.A. // J. Chem. Phys., 1956, vol. 24, p. 966.

3. Hush N.S. // J. Chem. Phys., 1958, vol. 28, p. 962.

4. Levich V.G. Kinetics of Reactions with Charge Transfer. Physical Chemistry, an Advanced Treatise, vol. Xb, ed. by Eyring H., Henderson D., Jost W. New York, Academic Press, 1970.

5. Grote R.F., Hynes J.T. // J. Chem. Phys., 1980, vol. 73, p. 2715.

6. Morgan J.D., Wolynes P.G. // J. Phys. Chem., 1987, vol. 91, p. 874.

7. Bixon M., Jortner J.J. // FEBS Lett., 1986, vol. 200, p. 303.

8. Sumi H., Marcus R.A. // J. Chem. Phys., 1987, vol. 86, p. 2096.

9. Mohr J.H., Schmickler W. // Phys. Rev. Letters, 2000, vol. 84, p. 1051.

10. van Kampen N.G. Stochastic Processes in Physics and Biology, Amsterdam, North Holland, 1981.

11. Landau L.D., Lifshitz E.M. Quantum Mechanics. Oxford, Pergamon, 1965.

12. Zener C. // Proc. Roy. Soc., 1932, vol. 137, p. 696.

13. Szabo A., Schulten K., Schulten Z. // J. Chem. Phys., 1990, vol. 72, p. 4350.

14. Deutch J.M. // J. Chem. Phys., 1980, vol. 73, p. 4700.

15. Kramers H.A. // Physica, 1940, vol. 7, p. 84.

16. Hänggi P., Talkner P., Borkovec M. // Rev. Mod. Phys., 1990, vol. 62, p. 251.

17. Schmickler W. // J. Electroanal. Chem., 1986, vol. 204 p. 31.

18. Kuznetsov A. M. Stochastic and Dynamic Views of Chemical Reactions in Solutions. Lausanne, Presses Polytechniques et Universitaires Romandes, 1999, p. $68 \mathrm{ff}$.

19. Zusman L.D. // Chem. Phys., 1980, vol. 49, p. 295. 


\section{Ступінь реакцій електронного переносу в дифузійній границі}

\section{В.Шміклер}

Університет Ульму, D-89069, Ульм, Німеччина

Отримано 8 серпня 2001 р., в остаточному вигляді - 10 жовтня $2001 \mathrm{p}$.

Проста реакція електронного переносу розглядається в дифузійній границі, в якій рух розчинника описується рівнянням Смолуховського. Імовірність електронного переходу розраховується з виразу Ландау-Зенера. Константа реакції розрахована як функція сили електронної взаємодії між реактантами. Для слабких взаємодій ступінь реакції співпадає з виразом, отриманим в першому наближенні теорії збурень. Для сильних взаємодій динаміка розчинника визначається ступенем реакції. Представлені розрахунки поєднують обидві границі.

Ключові слова: реакції електронного переносу, дифузійна границя

PACS: 82.30.F, 82.20.Gk 